\title{
EXPERIÊNCIAS DO OBSERVATÓRIO EM EDUCAÇÃO ESPECIAL E INCLUSÃO ESCOLAR: EM FOCO AS PRÁTICAS CURRICULARES E A FORMAÇÃO DE PROFESSORES ${ }^{1}$
}

\author{
PLETSCH, Márcia Denise ${ }^{2}$ \\ DE OLIVEIRA, Mariana Corrêa Pitanga ${ }^{3}$ \\ LIMA, Marcela Francis Costa ${ }^{3}$
}

\section{RESUMO}

Este texto apresenta as práticas e pesquisas desenvolvidas no âmbito do grupo Observatório da Educação Especial e inclusão escolar: práticas curriculares e processos de ensino e aprendizagem, sobretudo a partir de 2008, com a implementação da Política Nacional de Educação Especial na Perspectiva da Educação Inclusiva e das Diretrizes Operacionais do Atendimento Educacional Especializado na Educação Básica, modalidade Educação Especial. A análise focará, a partir de pesquisa de campo na perspectiva qualitativa, os impactos dessas mudanças nas práticas pedagógicas dos professores e a sua formação, assim como no desenvolvimento dos alunos com necessidades educacionais especiais. Os resultados evidenciaram, entre outros aspectos, a diversidade de propostas adotadas entre as redes de uma mesma região. Igualmente, ilustraram os desafios vivenciados pelos professores na garantia de tais diretrizes.

PALAVRAS-CHAVE: Inclusão escolar - Práticas pedagógicas - Formação de professores.

\footnotetext{
${ }^{1}$ Este texto apresenta resultados de pesquisas financiadas pelo Programa Observatório da Educação da CAPES, pelo CNPQ e pela FAPERJ. Os resultados foram apresentados pelas autoras no I Seminário Internacional de Inclusão Escolar: práticas em diálogo, realizado nos dias 21, 22 e 23 de outubro de 2014. Uma versão ampliada dessa discussão foi publicada por Pletsch (2014) na revista Poíesis Pedagógica.

2 Professora Adjunta do Departamento Educação e Sociedade e do Programa de Pós-Graduação em Educação, Contextos Contemporâneos e Demandas Populares (PPGEduc) da Universidade Federal Rural do Rio de Janeiro (UFRRJ). Jovem Cientista do Nosso Estado da FAPERJ. E-mail: marciadenisepletsch@gmail.com

3 Mestranda em Educação do Programa de Pós-Graduação em Educação, Contextos Contemporâneos e Demandas Populares (PPGEduc/UFRRJ) com bolsa da CAPES/OBEDUC.
} 
e-Mosaicos - Revista Multidisciplinar de Ensino, Pesquisa, Extensão e Cultura do Instituto de Aplicação Fernando Rodrigues da Silveira (CAp-UERJ)

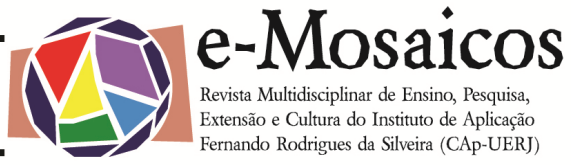

\section{ABSTRACT}

This paper presentes practices and researches carried out under the Observatory in Special Education and School Inclusion: curricular practices and processes of teaching and learning, especially from 2008 with the implementation of the National Politic on Special Education and Inclusive Education in the perspective of the Inclusion and of the Operational Guidelines of the Educational Service Specialist in Basic Education, Special Education mode. The analysis will focus, from field research, on qualitative perspective, the impacts of these changes in pedagogical practices of teachers and their training, as well as on the development of pupils with special educational needs. The results showed, among other aspects, the diversity of proposals adopted between network of the same locality. It also illustrated the challenges experienced by teachers in order to guarantee these guidelines.

KeYWORDS: Pedagogical practices - School inclusion - Teacher training.

Com o avanço das políticas de educação inclusiva, sobretudo a partir de 2008, temos enfrentado novos desafios no contexto da escola para garantir a escolarização da diversidade do alunado, em particular do público-alvo da Educação Especial que envolve sujeitos com deficiências intelectual/mental e sensorial, transtornos globais do desenvolvimento e altas habilidades/superdotação (BRASIL, 2008, 2009).

Com base nos avanços legais, o grupo de pesquisa, registrado no Conselho Nacional de Desenvolvimento Científico e Tecnológico (CNPq), "Observatório de Educação Especial e inclusão escolar: práticas curriculares e processos de ensino e aprendizagem", vinculado ao Programa de Pós-Graduação em Educação, Contextos Contemporâneos e Demandas Populares (PPGEduc/ UFRRJ), tem se debruçado para analisar as dimensões que envolvem a implementação das políticas federais de inclusão escolar nas redes de ensino da Baixada Fluminense, Estado do Rio de Janeiro, região com uma população de aproximadamente quatro milhões de habitantes e composta por treze municípios.

Neste artigo, nossa proposta não é realizar mais uma discussão sobre a Política Nacional de Educação Especial na Perspectiva da Educação Inclusiva
(BRASIL, 2008) e as Diretrizes Operacionais do Atendimento Educacional Especializado na Educação Básica, modalidade Educação Especial (BRASIL, 2009), uma vez que já existem inúmeras análises sobre as dimensões políticas, econômicas e sociais que envolvem a implementação de tais documentos (PLETSCH, 2011, MENDES, SILVA \& PLETSCH, 2011; TARTUCI, 2012; BRAUN, 2012; GARCIA, 2013, KASSAR, 2013, entre outros). Nosso objetivo é sintetizar resultados sobre nossas investigações sobre os impactos dessas políticas nas práticas curriculares dos professores e como as mesmas têm impactado nos processos de escolarização de alunos público alvo da Educação Especial, em particular com deficiência intelectual e múltipla ${ }^{4}$, alvos prioritários em nossas pesquisas. Para tal, nossas investigações têm usado o referencial histórico-cultural baseado em Vigotski ${ }^{5}$.

\footnotetext{
${ }^{4}$ Sobre as especificidades e conceitos adotados em relação a esses sujeitos, sugerimos ver nossas discussões publicadas em Pletsch (2014a) e Rocha e Pletsch (2015).

${ }^{5} \mathrm{Em}$ termos nacionais é amplamente estudado por Smolka e colaboradores (2010, 2011, 2011a, 2013). Em nível internacional autores como Baquero (2001), Van Der Veer e Valsiner (2009) e Friedrich (2012) tem sido nossas principais referências.
} 
e-Mosaicos - Revista Multidisciplinar de Ensino, Pesquisa, Extensão e Cultura do Instituto de Aplicação Fernando Rodrigues da Silveira (CAp-UERJ)

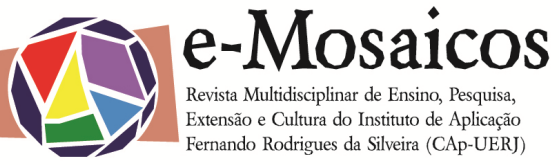

Os fundamentos metodológicos empregados em nossas pesquisas seguem os pressupostos qualitativos com base na pesquisa-ação e na abordagem etnográfica.As investigações que temos realizadas também se relacionam de forma profícua com a microgenética, abordagem metodológica conhecida como "análise microgenética" (GÓES, 2000). Em texto anterior (PLETSCH \& ROCHA, 2014), mostramos que, para além do vínculo com a teoria histórico-cultural, suas características e procedimentos vão ao encontro do perfil dos sujeitos participantes de nossas pesquisas, pois nos permite observar atentamente expressões faciais, pequenos gestos ou até mesmo ruídos que somente com a observação participante podem passar despercebidos.

Outro referencial metodológico empregado em nossas pesquisas é a pesquisa-ação, caracterizada como um método de investigação científica que tem como característica principal a participação ativa dos indivíduos pertencentes ao campo da pesquisa onde o projeto está sendo desenvolvido. Ao adotar essa proposta, o pesquisador deve estar aberto para conhecer e participar da dinâmica cotidiana da escola - os problemas, as necessidades e as prioridades -, e não trazer pressupostos teóricos prontos, sem levar em consideração a cultura local (BRAUN, 2004, 2012, 2013).

Após esta breve apresentação dos referenciais teórico-metodológicos que fundamentam nossos projetos, discutiremos os dados das três pesquisas desenvolvidas no período de 2009 até a atualidade.

O primeiro projeto $(2009-2012)^{6}$ objetivou analisar a implementação e

\footnotetext{
6 Projeto de Pesquisa "Observatório de políticas públicas em Educação Especial e inclusão escolar: estudo sobre as políticas públicas em Educação
}

operacionalização das políticas federais de inclusão escolar, bem como conhecer a forma pela qual 0 atendimento educacional especializado previsto nas atuais diretrizes vem sendo oferecido em nove dos treze municípios da Baixada Fluminense. Os resultados evidenciaram as contradições, dificuldades e estratégias usadas por essas redes de ensino para implementar tais políticas, sobretudo no que se refere à escolarização e ao atendimento educacional especializado dirigido para alunos com deficiência intelectual e condições mais acentuadas de desenvolvimento. Ficou claro também que, apesar da existência de diretrizes federais, as redes municipais têm implementado ações muito diversas entre si a partir da sua realidade social e das demandas locais. Dentre os principais resultados destacamos que: A) Alunos com deficiência intelectual ou múltipla não estão desenvolvendo processos de ensino e aprendizagem que garantam o efetivo desenvolvimento; B) Muitos alunos com deficiência intelectual, por não terem sido alfabetizados, acabam sendo encaminhados para classes de Educação de Jovens e Adultos (EJA) - "EJA especial". C) As redes, de maneira geral, apresentam falta de clareza sobre a avaliação e identificação desses sujeitos, o que pode impactar na distribuição de recursos, mas, sobretudo, no encaminhamento e nos suportes educacionais oferecidos a esses alunos.

\section{Especificamente sobre 0} atendimento educacional especializado nas salas de recursos multifuncionais, os dados sinalizaram para a: falta de estrutura física nas escolas para implementar as salas de recursos; problemas com a instalação do material

Especial e inclusão escolar de alunos com necessidades educacionais especiais nos municípios da Baixada Fluminense", financiado pelo CNPq, Processo 400548/2010-0. 
e-Mosaicos - Revista Multidisciplinar de Ensino, Pesquisa, Extensão e Cultura do Instituto de Aplicação Fernando Rodrigues da Silveira (CAp-UERJ)

distribuído pelo Ministério da Educação; falta de acessibilidade arquitetônica e ausência ou precariedade de transporte adaptado; salas de recursos multifuncionais superlotadas; falta de clareza dos profissionais sobre como realizar o trabalho colaborativo entre 0 professor do AEE com o professor da turma comum de ensino; falta de profissionais especializados para atuar no $A E E$, bem como falta de formação continuada, dentre outros.

A segunda pesquisa realizada no âmbito do grupo de pesquisa ${ }^{7}$,desenvolvida em rede com a parceria de três Programas de PósGraduação em Educação (UFRRJ, UDESC e UNIVALI), analisa as dimensões que envolvem a escolarização de alunos com deficiência intelectual, especialmente as que se referem ao ensino e aprendizagem destes nas classes regulares (ensino fundamental e educação de jovens e adultos), no atendimento educacional especializado e ao seu consequente desempenho nas avaliações nacionais em termos de rendimento escolar. 0 projetoobjetiva ainda, entre outros aspectos, examinar os caminhos cognitivos desenvolvidos por estes sujeitos na construção de conceitos científicos prioritariamente desenvolvidos no espaço formal de educação. Trata-se de um estudo longitudinal a ser realizado no período de março de 2013 a março de 2017.

Os resultados preliminares, especificamente das redes de ensino participantes do Estado do Rio de Janeiro, apontam inúmeras e diferentes situações

\footnotetext{
7 Projeto de Pesquisa "Observatório da Educação - A escolarização de alunos com deficiência intelectual: políticas públicas, processos cognitivos e avaliação da aprendizagem", financiado pelo Observatório da Educação da CAPES e do edital Apoio a projetos de pesquisa na área de humanidades/ 2011 (Proc. n. ${ }^{\circ}$ 110.061/2012).
}

de escolarização e suporte (ou não) educacional oferecido aos alunos com deficiência intelectual, assim como mostram as dificuldades das professoras para planejar estratégias de ensino e aprendizagem para esses sujeitos que promovam aos mesmos conceitos científicos entendidos aqui como necessários para o desenvolvimento de conhecimentos formais ensinados na escola. Nesse sentido, é importante ressaltar que os conceitos cotidianos (apontam 0 desenvolvimento real do sujeito) e os conceitos científicos (apontam para a zona de desenvolvimento proximal) se relacionam e se influenciam constantemente e, a partir das devidas mediações, promovem o aprendizado e, consequentemente, 0 desenvolvimento dos processos psicológicos superiores (PLETSCH, 2010), como por exemplo, a aprendizagem de conceitos relacionados ao ensino de matemática (a vista, a prazo, entre outros anteriores a estes, como, o significado da adição e subtração). Neste caso, verificamos também, a partir da análise das práticas observadas e registradas em vídeo, a falta de diretrizes oficiais e de clareza dos docentes para elaborar os enunciados dos exercícios a serem realizados pelos alunos. Tal aspecto acaba prejudicando não apenas os alunos com deficiência intelectual, mas a todos os alunos da turma.

A elaboração conceitual por parte de alunos com deficiência intelectual e outras dificuldades de aprendizagem requer do professor o uso de diferentes estratégias pedagógicas para apoiar e mediar o ensino. E o que denominamos de conhecimentos didáticos, os quais, muitas vezes, são menosprezados na formação inicial e continuada de professores com justificativas do tipo "não há receitas de bolo". Concordamos que o processo educacional é muito mais complexo do que ensinar e aplicar estratégias pedagógicas, mas não podemos negar que 
os docentes precisam de parâmetros teóricos e metodologias de ensino para realizar o trabalho pedagógico de forma efetiva. Isto é, faz-se necessário garantir,na formação inicial dos nossos discentes (futuros professores) e continuada de professores (daqueles que já atuam no magistério), fundamentos teóricos articulados com ações práticas, a fim de que tenham condições de impulsionar processos de ensino e aprendizagem criativos e significativos aos alunos com deficiências e aos demais.

Todavia, como já alertamos em texto anterior (PLETSCH, 2010), as possibilidades de apropriação dos conceitos científicos e da cultura pelas pessoas com deficiência intelectual especialmente aqueles que envolvem memória, criação, atenção, raciocínio lógico, interpretação, enfim, as operações simbólicas como um todo - dependem das interações estabelecidas entre professor e aluno ou aluno e aluno durante as práticas pedagógicas, bem como dos estímulos aos quais são expostos em seu contexto social e cultural. Portanto, para que as práticas escolares possibilitem aos alunos com deficiência intelectual desenvolver novas formas de funcionamento mental, as mesmas devem priorizar 0 ensino dos conceitos, seus significados e sentidos. Esse aspecto reforça a importância da mediação e das experiências de aprendizagem a que os sujeitos são expostos no ambiente escolar.

Em outras palavras, o modo como enxergamos o aluno e as expectativas que temos sobre ele acaba impactando nas práticas que dirigimos para eles e, consequentemente, no seu desenvolvimento. Dainêz (2012) ao contar as histórias/trajetórias escolares de Guilherme e André mostra e problematiza a relação entre o fazer pedagógico dirigido para esses alunos e as concepções sobre 0 seu desenvolvimento e as possibilidades encontradas pelos mesmos para "compensar" suas especificidades. Mas, é preciso ter clareza que para trilhar outros caminhos de desenvolvimento faz-se necessário oferecer ao sujeito com deficiência intelectual condições sociais e educacionais para tal. Outras vezes, é preciso oferecer, ainda, suportes da área da saúde, especialmente nos casos de maior comprometimento. Infelizmente, nossos dados indicaram que a estrutura pública de saúde oferecida paraa população da Baixada Fluminense é bastante precária frente às demandas. Além disso, histórica e culturalmente, o sistema de saúde brasileiro tem focado no diagnóstico e/ou na reabilitação das pessoas com deficiências, não atuando em sintonia com a área educacional para planejar ações e intervenções mais abrangentes e sistematizadas (PLETSCH, 2014).

A terceira pesquisa ${ }^{8}$ teve como público alvo sujeitos com múltiplas deficiências, em grande medida, não oralizados. O objetivo dessa pesquisa foi analisar o trabalho pedagógico realizado para esses alunos no atendimento educacional especializado oferecido na sala de recursos multifuncionais. Nessa direção, a partir da reflexão colaborativa com as professoras do AEE, discutimos, em encontros quinzenais,os conceitos de mediação e compensação a partir da teoria histórico-cultural, bem como os mesmos podem ou não favorecer a construção e internalização de conceitos científicos, seus sentidos e significados por parte dos alunos com múltiplas deficiências.

A investigação foi realizada em duas classes de atendimento educacional

8 Projeto de Pesquisa "A ESCOLARIZAÇÃO DE ALUNOS COM MÚLTIPLAS DEFICIÊNCIAS EM UMA ESCOLA PÚBLICA DA BAIXADA FLUMINENSE: formação de professores e processos de ensino e aprendizagem", financiado pela FAPERJ, Processo E26/112.261/2012. 
e-Mosaicos - Revista Multidisciplinar de Ensino, Pesquisa, Extensão e Cultura do Instituto de Aplicação Fernando Rodrigues da Silveira (CAp-UERJ)

especializado em uma rede de ensino da Baixada Fluminense. Sobre os problemas relacionados às práticas educativas, entre outras questões, os dados mostraram: a) dificuldades de infraestrutura, materiais e recursos adequados para atender às necessidades educacionais especiais; b) problemas relacionados ao transporte adaptado público e/ou escolar; c) falta de articulação do sistema educacional com o sistema de saúde; d) falta de conhecimentos específicos dos professores para efetivar atividades pedagógicas que promovam o desenvolvimento desses alunos.

Sobre a falta de conhecimentos, as entrevistas realizadas com as professoras indicam que o projeto impactou de forma positiva às práticas desenvolvidas pelas mesmas, pois auxiliou na identificação de recursos da área de Tecnologias Assistivas e, a partir desta, de Comunicação Alternativa e Ampliada. Além disso, a discussão sobre o conceito de mediação tem auxiliado no entendimento do papel dos recursos empregados nas práticas docentes ${ }^{9}$.

Vale mencionar que, apesar das garantias legais (BRASIL, 2008; 2009; 2011), as salas de recursos multifuncionais (pertencentes ao AEE) funcionavam e atendiam a alunos com comprometimentos graves, em sua maioria, muito mais com base nos esforços pessoais das professoras do que por políticas públicas dirigidas para esse atendimento. Ou seja, mesmo sendo multifuncionais as salas eram precárias e não atendiam necessidades e demandas mínimas. Fica a questão: Por que 0 distanciamento entre as diretrizes federais que mostram o AEE como solução para os problemas da "inclusão" e a realidade cotidiana das salas pesquisadas? A

\footnotetext{
9 Para nossos estudos nessa área temos como principais referências Pelosi (2008), Nunes, Quitério, Walter, Schirmer \& Braun ( 2011), Rocha (2014).
}

precariedade e/ou não funcionamento desse atendimento também foi evidenciada na pesquisa de doutorado de Souza (2013),realizada num município de São Paulo ao relatar a trajetória escolar de Alan.

Nos encontros quinzenais, a equipe de pesquisa discutiuo processo de ensino e aprendizagem desses alunos, à luz da teoria histórico cultural de Vigotski (1997, 2003), especialmente sobre o conceito de compensação a partir das mediações pedagógicas realizadas. Os dados mostraram que os sujeitos demandam práticas e ações relacionadas ao desenvolvimento de habilidades comunicativas, percepção e atividades da vida cotidianaque não se efetivam no espaço da sala de recursos multifuncionais. As professoras apontaram que a escola, assim como está organizada atualmente, pouco tem contribuído para o desenvolvimento desses sujeitos com múltiplas deficiências.

Como já indicamos em outra produção do grupo de pesquisa, é importante sinalizarmos que as políticas federais indicam que 0 atendimento educacional especializado (AEE) em salas de recursos multifuncionais deve ser oferecido no contra turno para os alunos que o frequentam. Contudo, os dados das pesquisasrealizadas no âmbito do nosso grupo,em diferentes redes de ensino, mostram que, em muitos casos, alunos com deficiências mais acentuadas e múltiplas, acabam, apesar da matrícula no ensino regular, frequentando somente a sala de recursos multifuncionais duas ou três vezes por semana durante uma hora. Segundo informado em entrevistas com gestores e professores, essa é uma das alternativas encontradas pelas redes para atender as diretrizes federais levando em consideração a falta de estrutura de suas escolas para garantir um atendimento minimamente adequado a estes alunos 
e-Mosaicos - Revista Multidisciplinar de Ensino, Pesquisa, Extensão e Cultura do Instituto de Aplicação Fernando Rodrigues da Silveira (CAp-UERJ)

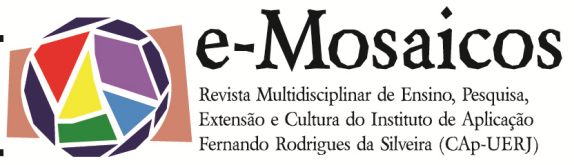

que demandam intervenções muito específicas (PLETSCH, 2014).

Sobre esses dados, temos nos questionado sobre qual seria o melhor espaço educacional para alunos com múltiplas deficiências, pois em dois dos casos analisados os sujeitos apresentam graves comprometimentos na linguagem (não oralizam), não possuem qualquer movimento motor, apenas teriam possibilidade em se comunicar (ou aprender a se comunicar) a partir do piscar dos olhos. Por exemplo, ao longo da análise dos vídeos e das observações participantes, não foi possível identificar o que um dos sujeitos estava sentindo, pois as expressões faciais eram as mesmas frente a todos os objetos e solicitações feitas pelas professoras.As docentes empregavam vários recursos para ensinálo, mas como eram apenas dois encontros semanais de uma hora cada, a intervenção não dava conta dos aspectos a serem desenvolvidos. Frente a estas questões as professoras evidenciaram que se sentem em vários momentos frustradas.

Para finalizar, nossas pesquisas têm revelado, entre inúmeras questões, as contradições, os desafios e as possibilidades encontradas pelos professores e gestores das diferentes redes de ensino para atender as políticas federais, a partir de suas realidades locais. Mas, para concluir, gostaríamos de dizer que precisamos ampliar o debate tão centrado nas políticas de educação inclusiva, para políticas que garantam, de fato e de direito, o acesso de todos os alunos com e sem deficiências aos processos de ensino e aprendizagem para uma vida cidadã, de forma a universalizar as riquezas sociais e culturais existentes. No caso dos sujeitos com comprometimentos graves, a garantia de intervenções pedagógicas precoces, assim como o oferecimento de uma rede de apoio estruturada, poderá possibilitar-Ihes interações sociais e educacionais que promovam um maior desenvolvimento.Por último, também registramos a demanda urgente por formações iniciais e continuadas mais vinculadas às demandas cotidianas dos professores em sala de aula, pois os dados nos indicaram que, em grande medida, as formações têm sido realizadas, mas sem dialogar com a realidade das escolas e sua dinâmica diária.

\section{REFERÊNCIAS BIBLIOGRÁFICAS}

BAQUERO. Ricardo. Vygostky $e$ a aprendizagem escolar. Tradução: Ernani F. da Fonseca Rosa Artes médicas: Porto Alegre, 1998.

BRASIL. Política Nacional de Educação Especial na perspectiva da Educação Inclusiva. Brasília, janeiro de 2008.

BRASIL.Diretrizes Operacionais para 0 Atendimento Educacional Especializado na Educação Básica, modalidade Educação Especial. Resolução 4. Brasília, 2009.

BRASIL. Decreto 7.611. Brasília, 2011.

BRAUN, Patricia. Análise quaseexperimental dos efeitos de um programa instrucional sobre autocontrole para professores da educação infantil e do ensino fundamental. Dissertação (Mestrado em Educação) - Programa de Pós-Graduação em Educação, Universidade do Estado do Rio de Janeiro, Rio de Janeiro, 2004.

BRAUN, Patricia. Uma intervenção colaborativa sobre os processos de ensino e aprendizagem do aluno com deficiência intelectual. Tese (Doutorado)

Universidade do Estado do Rio de Janeiro, 324 f., 2012.

BRAUN, Patricia.A pesquisa-ação: aspectos conceituais, aplicações e implicações em investigações sobre Educação Especial.In: 
NUNES, Leila Regina D'Oliveira. Novas trilhas o modo de fazer pesquisas em Educação Especial. Editora da ABPEE, São Paulo, 2014, 89-104..

DAINÊZ, Débora. Constituição humana, deficiência e educação: problematizando o conceito de compensação na perspectiva histórico cultural. 119f. Projeto de qualificação de doutorado (Educação), Universidade Estadual de CAMPINAS, Campinas, 2012.

FRIEDRICH, Janette. Lev Vigotski mediação, aprendizagem e desenvolvimento: uma leitura filosófica e epistemológica. Editora Mercado das Letras, São Paulo, 2012.

GARCIA, Rosalba Maria Cardoso. Política de educação especial na perspectiva inclusiva e a formação docente no Brasil. In: Revista Brasileira de Educação, v. 18, no 52, jan.-mar. 2013.

GÓES, Maria Cecília R.. A abordagem microgenética na matriz histórico-cultural: uma perspectiva para o estudo da constituição da subjetividade. Cadernos CEDES, v. 20, p. 9-25, 2000.

KASSAR, Mônica de Carvalho Magalhães \& SILVA, Fabiany de Cássia Tavares (orgs.). Educação e pesquisa no Centro-Oeste: processos de escolarização e práticas educativas. Editora UFMS, Campo Grande/MS, 2012, p. 227-257.

KASSAR, Mônica de Carvalho Magalhães. Uma breve história da Educação das pessoas com deficiência no Brasil. In: MELLETTI, Silvia Márcia Ferreira \& KASSAR, Mônica de Carvalho Magalhães (ogs.). Escolarização de alunos com deficiência: desafios e possibilidades.Editora Mercado das Letras, São Paulo, 2013, p. 33-76.
MENDES, Geovana Mendonça Lunardi; SILVA, Fabiany de C.Tavares ; PLETSCH, Márcia Denise. Atendimento educacional especializado: por entre políticas, práticas e currículo um espaçotempo de inclusão? In: Revista Contrapontos (UNIVALI),v. 11, p. 27-44, 2011.

NUNES, Leila Regina d'Oliveira de Paula; QUITÉRIO, Patricia Lorena; WALTER, Cátia de Figueiredo; SCHIRMER, Carolina Rizzotto; BRAUN, Patricia. Comunicar é preciso: em busca das melhores práticas na educação do aluno com deficiência. Editora ABPEE. São Paulo, 2011.

PELOSI,Miriam Bonadiu. Inclusão $e$ Tecnologia Assistiva. 2008. Volumes I e II, 303f. Tese (Doutorado em Educação) Programa de Pós-graduação em Educação da Faculdade de Educação, Universidade do Estado do Rio de Janeiro, Rio de Janeiro, 2008.

PLETSCH, Márcia Denise.Repensando a inclusão escolar: diretrizes políticas, práticas curriculares e deficiência intelectual. Rio de Janeiro: NAU/EDUR, 2010.

PLETSCH, Márcia Denise. A dialética da inclusão/exclusão nas políticas educacionais para pessoas com deficiências: um balanço do governo Lula (2003-2010). In: Revista Teias, v. 12, no. 24, p.39-55, Rio de Janeiro, jan./abril de 2011.

PLETSCH, Márcia Denise \& ROCHA, Maíra Gomes de Souza da. A etnografia como abordagem metodológica em pesquisas na Educação Especial. In: NUNES, Leila Regina D'Oliveira. Novas trilhas no modo de fazer pesquisas em Educação Especial.Editora da ABPEE, São Paulo, 2014, p 73-88.

PLETSCH, Márcia Denise. Educação Especial e inclusão escolar: políticas, 
e-Mosaicos - Revista Multidisciplinar de Ensino, Pesquisa, Extensão e Cultura do Instituto de Aplicação Fernando Rodrigues da Silveira (CAp-UERJ)

práticas curriculares e processos de ensino e aprendizagem. In: Revista Poíesis Pedagógica, v. 12, no 1, 2014.

PLETSCH, Marcia Denise. A escolarização de pessoas com deficiência intelectual no Brasil: da institucionalização às políticas de inclusão (1973-2013). In: RevistaArquivos Analíticos de Políticas Educativas, 22(81). Disponível em: http://epaa.asu.edu/epaa/v22n81.

Acessado em agosto de 2014a.

ROCHA, Maíra Gomes de Souza da. Processos de ensino e aprendizagem de alunos com múltiplas deficiências no atendimento educacional especializado à luz da teoria histórico-cultural. Dissertação (Mestrado em Educação), Programa de Pós-Graduação em Educação, Contextos Contemporâneos e Demandas Populares, UFRRJ, 2014.

ROCHA, Maíra Gomes de Souza da. e PLETSCH, Márcia Denise. Deficiência múltipla: disputas conceituais e políticas educacionais no Brasil. In: Revista Cadernos de Pesquisa da UFMA, v. 22, n01, jan./abr., 2015.

SOUZA, Flávia Faissal de. Políticas de educação inclusiva: análise das condições de desenvolvimento dos alunos com deficiência na instituição escolar. 297f. Tese (Doutorado em Educação), Universidade Estadual de Campinas, Campinas, 2013.

SMOLKA, Ana Luiza Bustamante \& NOGUEIRA, Ana Lúcia Horta (org.). Questões de desenvolvimento humano práticas e sentidos. Editora Mercado das Letras. São Paulo, 2010.

SMOLKA, Ana Luiza Bustamante \& NOGUEIRA, Ana Lúcia Horta (org.).Emoção, memória, imaginação - a constituição do desenvolvimento humano na história e na cultura. Editora Mercado das Letras. São Paulo, 2011.

SMOLKA, Ana Luiza Bustamante \& GÓES, Maria Cecília Rafael de (org.). A linguagem e o outro no contexto escolar: Vigotski e a construção do conhecimento. 14 edição. Papirus, Campinas, 2011a.

SMOLKA, Ana Luiza Bustamante \& NOGUEIRA, Ana Lúcia Horta (org.). Estudos na perspectiva de Vigotski: gênese e emergência das funçôes psicológicas.Editora Mercado das Letras. São Paulo, 2013.

TARTUCI, Dulcéria. Observatório goiano de Educação Especial: indícios da formação de professores e implementação do atendimento educacional especializado. In: VEER, René Van Der \& VALSIER, Jaan. Vygotsky - umasíntese.Editora Loyola, São Paulo, 2009.

VIGOTSKI, Lev Semenovich. Obras escogidas V. Visor. Madrid, 1997.

VIGOTSKI, Lev Semenovich. $A$ formação social da mente. Editora Martins Fontes, São Paulo, 2003.
Recebido em: 10/06/2015 Aceito em: 29/06/2015 